\title{
Fetal and Maternal Non-glucose Carbohydrates and Polyols Concentrations in Normal Human Pregnancies at Term
}

\author{
VALENTINA BRUSATI, MACIEJ JÓŹWIK, MARCIN JÓŹWIK, CECILIA TENG, CINZIA PAOLINI, \\ ANNA MARIA MARCONI, AND FREDERICK C. BATTAGLIA \\ Department of Obstetrics and Gynecology [V.B., C.L.P., A.M.M.], DMSD San Paolo Hospital, University \\ of Milan, 20142 Milan, Italy; Department of Gynecology [Mac J., Mar J.], Medical University of \\ Bialystok, 15-276 Bialystok, Poland; Departments of Obstetrics-Gynecology and Pediatrics [C.T., F.C.B.], \\ University of Colorado, Aurora, CO 80045
}

\begin{abstract}
The objective of the present investigation was to determine
fetal and maternal plasma concentrations of nonglucose carbo-
hydrates and polyols in normal human pregnancies at term.
Uncomplicated human pregnancies ( $n=50$ ) were studied at $\geq 37$
wk gestation. Blood samples were obtained from umbilical ar-
tery, umbilical vein, and maternal peripheral blood at the time of
elective cesarean section. Plasma concentrations of inositol,
glycerol, erythritol, sorbitol, and mannose were determined by
HPLC analysis. Differences between umbilical venous, umbilical
arterial, and maternal concentration were tested by the two-tailed
$t$ test for paired samples. Correlations between umbilical and
maternal concentration and between umbilical venoarterial con-
centration difference and umbilical arterial concentration were
assessed by Pearson's correlation and multiple regression anal-
ysis. All newborns were appropriate for gestational age, and
oxygenation and acid-base balance were within the normal range
\end{abstract}
The transport of glucose across the human placenta has been well studied in both normal and high-risk pregnancies (1-4). However, the study of the other carbohydrates and sugar alcohols in plasma has received far less attention. In part, this may reflect the untested assumption that other carbohydrates and polyols can be produced from glucose in sufficient amounts to meet fetal requirements. One might assume, then, that there is little need for a supply of these other compounds to the fetus by placental transport. However, studies of the nutritional supply of amino acids in early development have

Received November 30, 2004; accepted March 21, 2005.

Correspondence: Anna Maria Marconi, M.D., Ospedale San Paolo, Via A. di Rudini, 8, 20142 Milano, Italy; e-mail: annamaria.marconi@unimi.it.

This work was supported by March of Dimes Grant 1FY01-559 and National Institutes of Health Grant 2 RO1 HD34837-05A2 and General Clinical Research Center Grant MO1 RR00069.

Preliminary account of this work was presented at the 2001 Pediatric Academic Societies Annual Meeting; April 28-May 01, 2001; Baltimore, MD.

DOI: 10.1203/01.PDR.0000180549.86614.73 for all fetuses studied. For most of the polyols (inositol, sorbitol, and erythritol), the fetal concentration was significantly higher than the maternal concentration. The umbilical venoarterial concentration difference for inositol was $-10.5 \pm 3.6 \mu \mathrm{M}$, for glycerol was $10 \pm 1.7 \mu \mathrm{M}$, for sorbitol was $3.8 \pm 0.5 \mu \mathrm{M}(p<$ 0.001 ), and for mannose was $7.6 \pm 0.7 \mu \mathrm{M}$. There was a significant correlation between maternal concentration and umbilical venous concentration of mannose $\left(\mathrm{UV}_{\text {MAN }}=15.38+\right.$ $\left.0.69 \mathrm{M}_{\text {MAN }} ; R^{2}=0.46 ; p<0.001\right)$. These results indicate that in normal human pregnancies at term, inositol is produced by the fetus, sorbitol is produced by the placenta, and there is a significant umbilical uptake of mannose from the maternal circulation. (Pediatr Res 58: 700-704, 2005)

$\mathbf{R B C}$, red blood cell emphasized that many amino acids that are considered "nonessential" may be required in the dietary supply to the fetus or the newborn $(5,6)$. The same may be true of some carbohydrates. Recent studies have suggested that mammalian cells may require an exogenous supply of mannose to meet mannose 6-phosphate requirements for glycoprotein synthesis (7). Furthermore, the biologic importance of the polyols has received attention with respect to their accumulation in reproductive tissues $(8-10)$ and to their roles in complications of diabetes $(11,12)$. A recent study demonstrated that a low maternal serum inositol concentration is associated with a 2.6-fold increased risk for offspring with spina bifida (13). In addition, the increased risk for neural tube defects that is found in mothers with diabetes could be explained by a secondary cellular depletion of inositol as a result of an inhibited cellular uptake from hyperglycemia (13).

However, there have been few studies in human pregnancies of inositol and of sorbitol in terms of their maternal and fetal concentrations (14-17). For the rest of the sugars and sugar 
alcohols, their concentrations in maternal and fetal plasma are unknown. However, a recent study has shown that some of the polyols are in relatively high concentration in human embryonic fluids, which suggests an important biologic role during early development (18). The present study was directed at determining the normal maternal-fetal concentration differences for these compounds, as well as establishing whether there is any significant fetal or placental uptake of these sugars and alcohols.

\section{METHODS}

Pregnant women were enrolled for the study at two different sites: 1) the Department of Obstetrics and Gynecology, University of Milan, DMSD San Paolo (Milan, Italy), and 2) the Department of Gynecology, Medical University of Bialystok (Bialystok, Poland). The reason for the two sites is that the obstetricians in Milan and Bialystok had worked together in the Division of Perinatal Medicine at the University of Colorado and the collaborative research stemmed from this joint interaction. HPLC analyses were conducted at the Perinatal Research Facility of the University of Colorado School of Medicine (Aurora, CO). The protocol of the study was approved in advance by the Ethical Committee of the San Paolo Hospital and by the Ethical Committee of the Medical University of Bialystok. Informed consent was obtained from all pregnant women.

Patients. Fifty uncomplicated singleton pregnancies were studied at term ( $\geq 37 \mathrm{wk}$ of gestation) at the time of elective cesarean section. All cesarean sections were performed under peridural anesthesia. The indications for cesarean section were breech presentation, one or more previous cesarean sections, previous myomectomy, or placenta previa. Gestational age was calculated from the last menstrual period and confirmed by routine ultrasound within 20 wk of gestation. Immediately after delivery of the newborn, umbilical arteria and venous blood samples were obtained from a doubly clamped segment of the cord. Simultaneously, maternal peripheral blood was obtained from a radial artery or from an antecubital vein.

Analytical methods. Blood samples were collected into heparinized syringes or into preheparinized plastic tubes (Life Sciences, Denver, CO). Fetal $\mathrm{Hb}$ concentration, oxygen saturation $\left(\mathrm{O}_{2}\right.$ sat $)$, umbilical arterial $\mathrm{pH}, \mathrm{PO}_{2}$ and $\mathrm{PCO}_{2}$ were measured on a Radiometer ABL 700 analyzer (Radiometer, Copenhagen, Denmark) in Italy and on a Ciba Corning 278 Blood Gas System (Ciba Corning Diagnostics, Medfield, MA) in Poland. Within 10 min from collection of the blood samples, plasma for carbohydrates and polyol analyses were separated by centrifugation at $4{ }^{\circ} \mathrm{C}$ and frozen at $-70^{\circ} \mathrm{C}$ until the time of analysis.

Plasma concentrations were determined mainly because almost all previous studies of sugar and polyol concentrations had been carried out on plasma. However, to test whether there was rapid exchange between plasma and red blood cells (RBCs), we incubated human umbilical cord blood with $14 \mathrm{C}$ labeled substrates at $37^{\circ} \mathrm{C}$ for up to $20 \mathrm{~min}$. The latter time was taken as the maximum time beginning with obtaining a sample and separating the plasma and RBCs. Figure 1 presents the data for such a study and illustrates that there was no evidence of rapid exchange between RBCs and plasma.

HPLC analysis. Plasma concentrations of glucose, inositol, glycerol, erythritol, sorbitol, and mannose were determined as previously described (19). Plasma was quickly thawed, and $0.1 \mathrm{~mL}$ of plasma was deproteinized by adding $0.1 \mathrm{~mL}$ of $0.3 \mathrm{~N}$ zinc sulfate that contained $30 \mathrm{mg} / 100 \mathrm{~mL}$ xylitol as internal standard and mixed well. A total of $0.1 \mathrm{~mL}$ of $0.3 \mathrm{~N}$ barium hydroxide then was added and mixed. After centrifugation at $14,000 \times g$ for $10 \mathrm{~min}$, the supernatant was filtered through a Millipore (Bedford, MA) filter (pore size $0.45 \mu \mathrm{m}$ ) and loaded on a refrigerated autosampler of a Dionex HPLC (Dionex Corp., Sunnyvale, CA). The separation of hexoses and polyols was carried out on a CarboPac MA1 anion-exchange column. The analysis was run isocratically with $500 \mathrm{mM}$ sodium hydroxide for $13 \mathrm{~min}$, followed by a step change to $300 \mathrm{mM}$ sodium hydroxide for $20 \mathrm{~min}$ at ambient temperature. The flow rate was $0.4 \mathrm{~mL} / \mathrm{h}$. All of the peaks were quantified using a pulse amperometric detector with a gold working electrode. The Dionex PeakNet software was used for instrument operation and data analysis. The concentrations of each sugar were calculated by using the integrated area under the peak. The internal xylitol standard was used to correct for instrument variances. All concentrations were expressed in $\mu \mathrm{M}$. Figure 2 presents chromatograms of maternal and fetal plasma achieved with this method using xylitol as an internal standard.

Calculations and statistical analysis. Umbilical venoarterial concentration differences for carbohydrates and polyols were determined as Umb diff $(\mu \mathrm{M})$ $=\mathrm{UV}-\mathrm{UA}$, where UV and UA are umbilical venous and arterial plasma

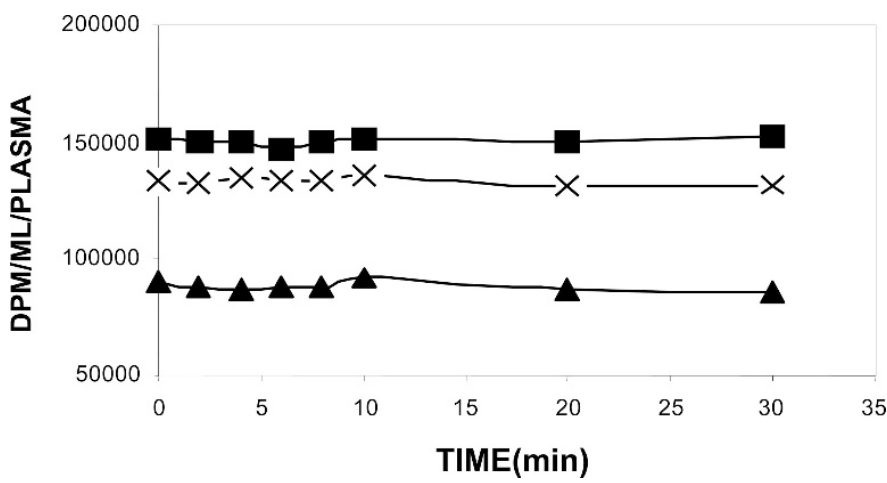

Figure 1. Exchange of nonglucose carbohydrates and polyols between RBCs and plasma. Human umbilical cord blood was incubated with 14C-labeled substrates at $37^{\circ} \mathrm{C}$ for up to $20 \mathrm{~min}$. A sample was obtained at different time intervals, plasma was separated, and radioactivity was measured. The steadystate curve shows that there is no evidence of rapid exchange of nonglucose carbohydrates and polyols between RBCs and plasma. $\square$, Inositol; $\boldsymbol{\Delta}$, mannose; X, sorbitol.
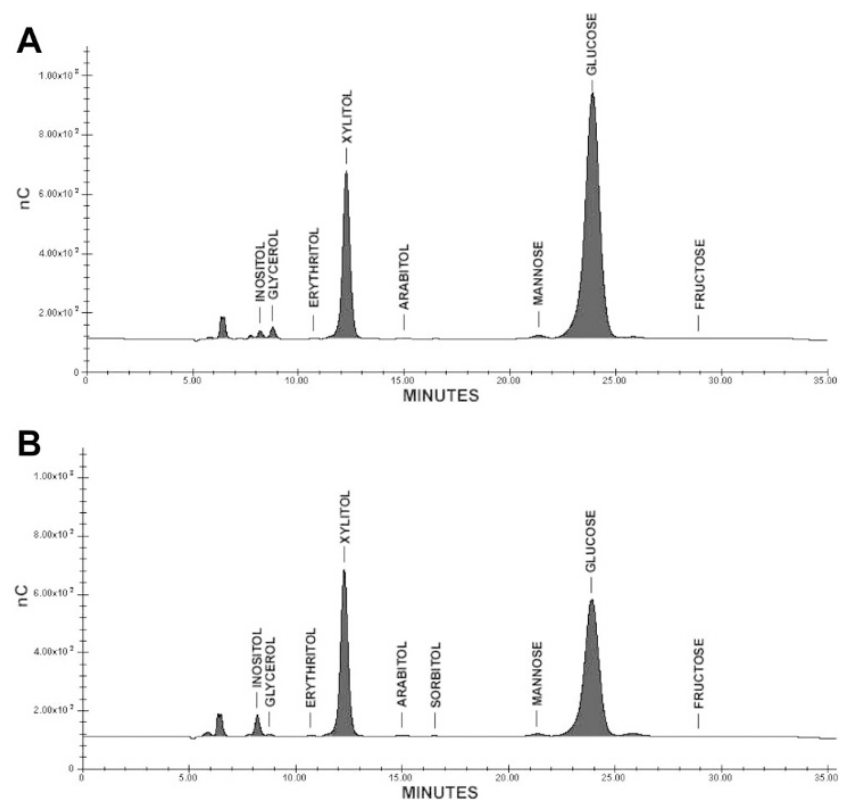

Figure 2. HPLC chromatograms of maternal $(A)$ and fetal $(B)$ plasma depicting the peaks for the various sugars and polyols are presented. The much higher peak for inositol in fetal blood is clearly evident in a comparison of the two figures. Xylitol is included as an internal standard.

concentrations. Fetal-maternal concentration ratios were calculated as $\mathrm{F} / \mathrm{M}=$ $\mathrm{UA} / \mathrm{M}$, where UA is the umbilical arterial plasma concentration and $\mathrm{M}$ is the maternal plasma concentration.

Data are presented as mean \pm SEM. An $F$ test first was performed on all data. Differences between the Italian and the Polish concentration data were tested using the $t$ test for unpaired samples. Differences between umbilical venous, umbilical arterial, and maternal concentrations were analyzed for significance with the two-tailed $t$ test for paired samples.

Correlation between umbilical and maternal concentrations and between umbilical concentration difference and umbilical arterial or venous concentrations were assessed by Pearson's correlation and multiple regression analysis. A $p<0.05$ was considered significant.

\section{RESULTS}

A total of 28 patients were enrolled in Italy, and 22 were enrolled in Poland. Characteristics of the studied population are presented in Table 1. All newborns were appropriate for 
Table 1. Clinical characteristics of the studied population

\begin{tabular}{|c|c|c|c|}
\hline & Italy & Poland & $\mathrm{p}$ \\
\hline Patients number & 28 & 22 & \\
\hline Gestational age (weeks) & $38.2 \pm 0.1$ & $38.9 \pm 0.4$ & ns \\
\hline Fetal weight $(\mathrm{g})$ & $3219 \pm 83$ & $3325 \pm 77$ & ns \\
\hline Placental weight (g) & $482 \pm 20$ & - & - \\
\hline Fetal $\mathrm{Hb}(\mathrm{g} / \mathrm{dl})$ & $14.5 \pm 0.3$ & $14.1 \pm 0.3$ & ns \\
\hline UA pH & $7.303 \pm 0.007$ & $7.283 \pm 0.010$ & ns \\
\hline $\mathrm{UA} \mathrm{pO}{ }_{2}(\mathrm{mmHg})$ & $17.9 \pm 1.1$ & $15.5 \pm 0.07$ & ns \\
\hline $\mathrm{UA} \mathrm{pCO}(\mathrm{mmHg})$ & $51.9 \pm 1.1$ & $54.6 \pm 1.8$ & ns \\
\hline
\end{tabular}

gestational age according to Italian (20) and Polish (21) birth weight-gestational age standards; oxygenation and acid-base balance were within the normal range for all of the studied fetuses according to data previously published (22).

Population differences. The differences for the data between the Italian and the Polish population were confined to the following maternal concentrations of inositol (Italian: $21.36 \pm$ $1.6 \mu \mathrm{M}$; Polish: $30.72 \pm 2.89 \mu \mathrm{M} ; p<0.01)$ and mannose (Italian: $60.51 \pm 1.90 \mu \mathrm{M}$; Polish: $73.03 \pm 3.90 \mu \mathrm{M} ; p<$ 0.01 ) and to umbilical venous concentrations of inositol (Italian: $60.76 \pm 3.21 \mu \mathrm{M}$; Polish: $72.62 \pm 4.83 \mu \mathrm{M} ; p<0.05)$ and sorbitol (Italian: $13.82 \pm 0.96 \mu \mathrm{M}$; Polish: $10.09 \pm 1.34$ $\mu \mathrm{M} ; p<0.05)$. In contrast, umbilical venoarterial concentration differences and fetal-maternal concentration ratios were not significantly different between the Italian and the Polish data for all carbohydrates. In addition, the Polish and Italian data were analyzed separately to confirm that significant umbilical venoarterial concentration difference, significant fetalmaternal concentration ratio, and significant relationships found in the pooled data were also found in the data analyzed separately in the two sites. Therefore, the results from the two groups have been pooled for analysis of these variables. Because, in the adult, there are no appreciable arteriovenous concentration differences for all of the carbohydrates and polyols that we considered, maternal arterial and venous samples have been pooled together.

Fetal and maternal concentrations. Umbilical venous, umbilical arterial, and maternal plasma concentrations of glucose, nonglucose carbohydrates, and polyols are presented in Table 2. Umbilical venous concentrations were significantly higher than maternal concentrations for inositol $(p<0.001)$, erythritol $(p<0.001)$, and sorbitol $(p<0.001)$, whereas maternal concentrations were significantly higher than umbilical venous concentrations for glycerol $(p<0.001)$ and mannose $(p<$ 0.01 ). Figure 3 presents the fetal-maternal ratios for the carbohydrates and polyols.
Umbilical venoarterial differences. Umbilical venoarterial concentration differences for nonglucose carbohydrates and polyols are presented in Fig. 4. There was a significant negative umbilical venous-arterial concentration difference for inositol $(-10.5 \pm 3.6 \mu \mathrm{M})$, suggesting fetal production of inositol. Figure 4 also shows that, on the contrary, umbilical venous concentration was significantly higher than arterial for glycerol, sorbitol, and mannose.

There was a significant correlation between the umbilical venous concentration and the maternal concentration of mannose (Fig. 5). Furthermore, there was a significant correlation between the umbilical arterial and the umbilical venous concentration of mannose $\left(\mathrm{UV}_{\mathrm{MAN}}=-2.53+0.91 \mathrm{UA}_{\mathrm{MAN}} ; R^{2}\right.$ $=0.89 ; p<0.001)$ but none between the umbilical venoarterial concentration difference of mannose and the umbilical venous concentration. Similarly, there was no significant correlation of umbilical venous mannose concentration with either the maternal or the umbilical venous concentration of glucose.

\section{DISCUSSION}

There have been many studies of the transplacental concentration gradient for glucose in human pregnancies and in animal studies $(1-4,19)$. These studies have also determined the relationship between glucose delivery to the fetus and the maternal glucose concentration. However, there have been virtually no comparable studies for the other carbohydrates, including the polyols. This is surprising given that there has been increasing evidence of the importance of nonglucose carbohydrates and polyols for human tissues. For example, studies in patients with diabetes have focused on the role of changes in sorbitol and inositol tissue concentrations as causative factors in the diabetic complications of cataracts and peripheral neuropathies, and individuals with diabetes have been shown to have higher tissue sorbitol concentrations and lower inositol concentrations compared with those without diabetes $(10,11)$. A patient with congenital cataracts has been shown to have absent sorbitol dehydrogenase activity in the lens. Transgenic mice expressing the aldose reductase gene, which catalyzes the production of sorbitol from glucose, develop susceptibility to diabetic and galactose cataracts (23).

Our study provides the first evidence of in vivo umbilical uptake of sorbitol by the human placenta. Production of sorbitol by the human placenta has been shown in vitro by Mango et al. (9) and Grimshaw and Lai (24). It is likely that the umbilical uptake reflects placental production, although without tracer studies and/or uterine arteriovenous concentration

Table 2. Concentrations of carbohydrates and polyols in maternal peripheral blood (M), umbilical vein (UV) and umbilical artery (UA)

\begin{tabular}{lccrr}
\hline & M & UV & UA & P (MvsUA) \\
\hline Glucose & $4553.1 \pm 100.8$ & $3590.5 \pm 82.6$ & $2942.1 \pm 81.4$ & $<0.001$ \\
Inositol & $25.74 \pm 1.75$ & $64.42 \pm 2.5$ & $75.43 \pm 3.6$ & $<001$ \\
Glycerol & $127.35 \pm 15.17$ & $33.48 \pm 1.91$ & $23.5 \pm 1.83$ & $<0.001$ \\
Erythritol & $7.74 \pm 1.02$ & $11.19 \pm 1.02$ & $12.64 \pm 1.20$ & $<.001$ \\
Sorbitol & $2.9 \pm 0.53$ & $12.23 \pm 0.86$ & $8.36 \pm 0.70$ & $<0.001$ \\
Mannose & $66.37 \pm 2.28$ & $60.73 \pm 2.23$ & $53.09 \pm 2.16$ & $<0.001$ \\
\hline
\end{tabular}

Concentrations are expressed in $\mu \mathrm{M}$. Values are mean \pm SE. p-Values were determined by Student's t-test for paired observations for maternal and umbilical venous plasma concentrations. 


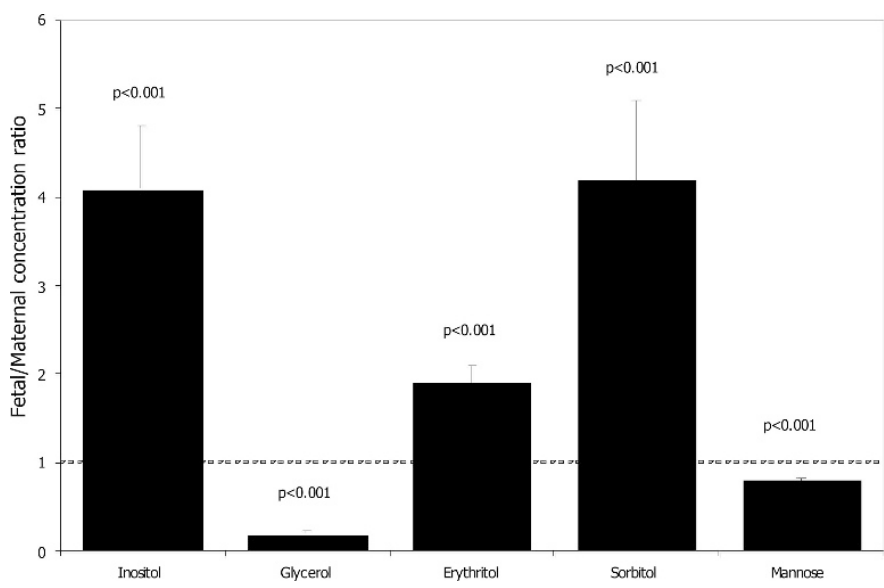

Figure 3. Fetal-maternal concentration ratios (F/M) of nonglucose carbohydrates and polyols in human pregnancies at term (mean $\pm \mathrm{SE})$. Significances of the difference of the ratio from 1 are presented.

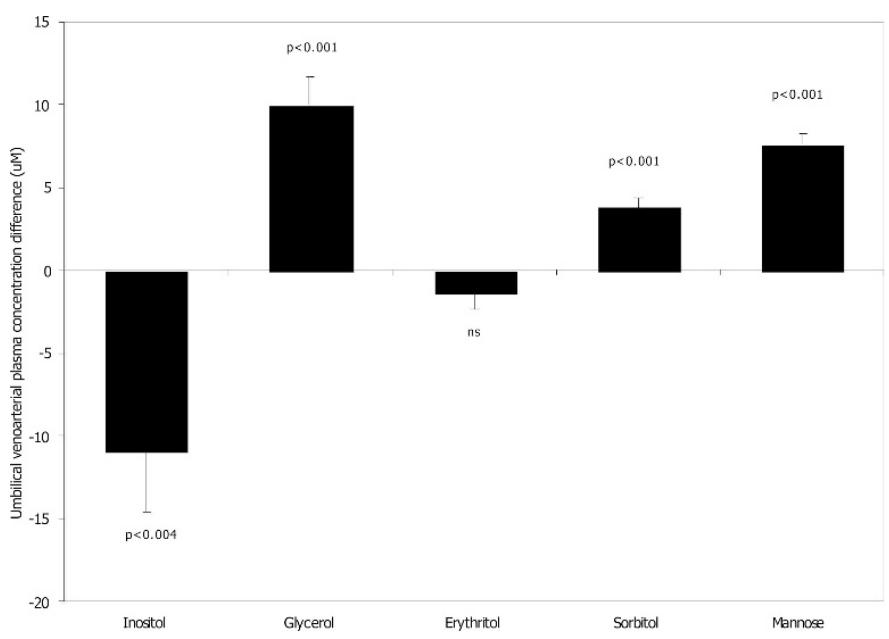

Figure 4. Umbilical venoarterial concentration differences of nonglucose carbohydrates and polyols in human pregnancies at term. Bar graphs are mean \pm SE. $p$ values for the concentration differences from 0 are presented.

differences, we cannot exclude the unlikely possibility of transport of this straight-chain alcohol from the maternal circulation. In the present study, no correlation could be found between maternal glucose concentration and the umbilical uptake of sorbitol.

Inositol concentrations are known to be elevated during fetal life and to decrease postnatally $(12,13)$. Free inositol is present in all tissues but is especially high in tissues whose cells do not divide rapidly in adult life: CNS, skeletal muscle, and cardiac muscle (12). The concentrations of inositol in these tissues are very high at the earliest stages of development $(12,13)$. The enzyme required for the synthesis of inositol from glucose (glucose-6-P cyclase) has been found in the human placenta and umbilical cord $(9,14)$. However, nothing is known about whether fetal inositol requirements are met by placental transport or by fetal production. The present study clearly establishes the importance of fetal production because there is no significant umbilical uptake of inositol into the fetal circulation despite the potential for placental production of inositol from glucose.

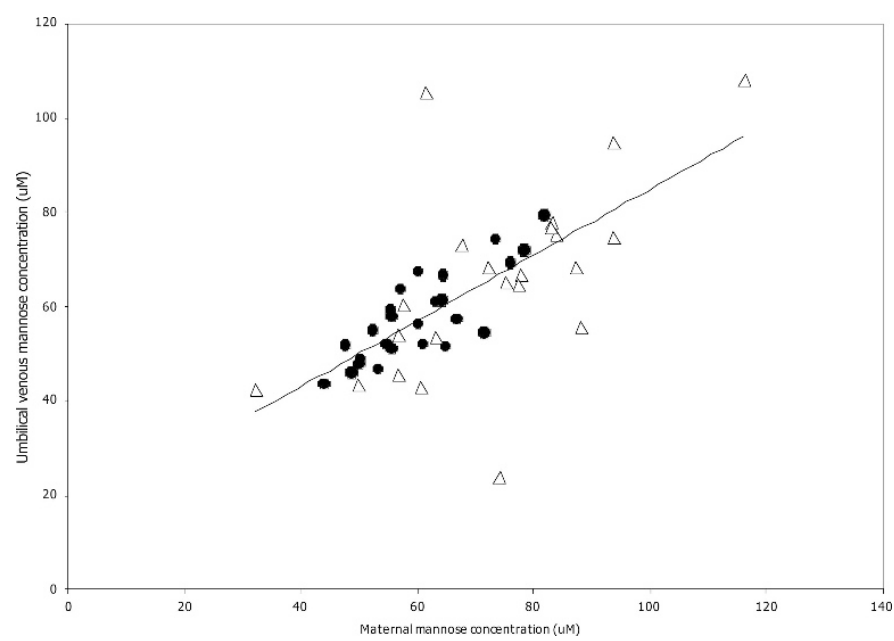

Figure 5. Relationship between umbilical venous plasma mannose concentration and maternal plasma mannose concentration: $\mathrm{UV}_{\mathrm{MAN}}=15.38+0.69$ $\mathrm{M}_{\mathrm{MAN}} ; R^{2}=0.46 ; p<0.001$., Italian data; $\triangle$, Polish data.

The present study has documented a surprising finding for mannose. This sugar is required for glycoprotein and glycophospholipid synthesis, and it can be synthesized from glucose. Given its very low concentration in maternal blood, it was logical to assume that fetal mannose requirements, like inositol requirements, are met by fetal synthesis rather than by placental transport. However, the present study establishes that in normal human pregnancies, there is a significant umbilical uptake of mannose. Recent in vitro studies have brought out two important new findings about mannose metabolism in human tissues. One set of studies documented the presence of a specific high-affinity mannose transporter in human fibroblasts (25). This transporter is capable of transporting mannose into cells even at the low mannose concentrations present in plasma. The characteristics of the transporter are distinct from glucose transporters in that the mannose transporter has a high affinity for mannose and a relatively low affinity for glucose. Furthermore, the study of Ogier-Denis et al. (26) showed that the mannose transporter is developmentally regulated in enterocytes. Our data demonstrating a significant umbilical uptake of mannose at relatively low maternal concentrations strongly suggest the presence of a mannose transporter in human trophoblast. A second set of studies (7) showed that the production of mannose glycans in human fibroblasts may require the presence of mannose rather than its intracellular production from glucose. Because mannose glycans accumulate in fetal tissues during development, the umbilical uptake of mannose, shown for the first time in the present study, is consistent with the requirement of an external supply of mannose to the fetus.

Finally, that polyol concentrations are elevated sufficiently in fetal blood to lead to the establishment of relatively large fetal-maternal concentration gradients for polyols such as inositol, sorbitol, and erythritol (Table 2) suggests that the trophoblast may be relatively impermeable to these compounds. The presence of large fetal-maternal concentration ratios for the polyols (Fig. 3) also suggests that the reduction of sugars to their corresponding alcohols is favored. The role of the polyols in developing tissues is currently unknown. 
Glycerol is known to be taken up across the placenta. The present study demonstrates the significant venoarterial concentration difference of glycerol and also shows that glycerol concentration gradient is from the maternal circulation to the fetal circulation. This is in contrast to the other polyols discussed above. Whether the nonglucose carbohydrates have altered metabolism in such diseases as maternal diabetes or fetal growth restriction is also unknown and will require further study.

\section{REFERENCES}

1. Stembera ZK, Hodr J 1966 The relationship between the blood levels of glucose, lactic acid and pyruvic acid in the mother and in both umbilical vessels of the healthy fetus. Biol Neonate 10:227-238

2. Bozzetti P, Ferrari MM, Marconi AM, Ferrazzi E, Pardi G, Makowski EL, Battaglia FC 1988 The relationship of maternal and fetal glucose concentrations in the human from midgestation until term. Metabolism 37:358-363

3. Nicolini U, Hubinont C, Santolaya J, Fisk NM, Coe AM, Rodeck CH 1989 Maternalfetal glucose gradient in normal pregnancies and in pregnancies complicated by alloimmunization and fetal growth retardation. Am J Obstet Gynecol 161:924-927

4. Marconi AM, Paolini C, Buscaglia M, Zerbe G, Battaglia FC, Pardi G 1996 The impact of gestational age and fetal growth on the maternal-fetal glucose concentration difference. Obstet Gynecol 87:937-942

5. Regnault TR, Battaglia FC 2003 Amino acid nutrition in utero: placental function and metabolism. In: Walker WA, Watkins JB, Duggan C (eds) Nutrition in Pediatrics, 3rd Ed. BC Decker Inc., Hamilton, pp 471-490

6. Ziegler EE, Fomon SJ, Carlson SJ 2003 The term infant. In: Walker WA, Watkins JB, Duggan C (eds) Nutrition in Pediatrics, 3rd Ed. BC Decker Inc., Hamilton, pp 515-527

7. Panneerselvam K, Etchison JR, Freeze HH 1997 Human fibroblasts prefer mannose over glucose as a source of mannose for $\mathrm{N}$-glycosylation. Evidence for the functional importance of transported mannose. J Biol Chem 272:23123-23129

8. Lewin LM, Yannai Y, Melmed S, Weiss M 1982 myo-Inositol in the reproductive tract of the female rat. Int J Biochem 14:147-150

9. Mango D, Scirpa P, Menini E 1976 Effects of dehydroepiandrosterone and 16 alpha-hydroxydehydroepiandrosterone on the reduction of glucose to glucitol by the human placenta. Horm Metab Res 8:302-307
10. Burton LE, Wells WW 1974 Studies on the developmental pattern of the enzymes converting glucose 6-phosphate to myo-inositol in the rat. Dev Biol 37:35-42

11. Jedziniak JA, Chylack LT Jr, Cheng HM, Gillis MK, Kalustian AA, Tung WH 1981 The sorbitol pathway in the human lens: aldose reductase and polyol dehydrogenase. Invest Ophthalmol Vis Sci 20:314-326

12. Lee AY, Chung SS 1999 Contributions of polyol pathway to oxidative stress in diabetic cataract. FASEB J 13:23-30

13. Groenen PM, Peer PG, Wevers RA, Swinkels DW, Franke B, Mariman EC, SteegersTheunissen RP 2003 Maternal myo-inositol, glucose, and zinc status is associated with the risk of offspring with spina bifida. Am J Obstet Gynecol 189:1713-1719

14. Battaglia FC, Meschia G, Blechner JN, Barron DH 1961 The free myo-inosito concentration of adult and fetal tissues of several species. Q J Exp Physiol Cogn Med Sci 46:188-193

15. Quirk JG Jr, Bleasdale JE 1983 myo-Inositol homeostasis in the human fetus. Obstet Gynecol 62:41-44

16. Toh N, Inque T, Tanaka H, Kimoto E 1987 Polyol accumulation in human placenta and umbilical cord. Biol Res Pregnancy Perinatol 8:13-15

17. Brachet EA 1973 Presence of the complete sorbitol pathway in the human normal umbilical cord tissue. Biol Neonate 23:314-323

18. Jauniaux E, Hempstock J, Teng C, Battaglia FC, Burton GJ 2005 Polyol concentrations in the fluid compartments of the human conceptus during the first trimester of pregnancy: maintenance of redox potential in a low oxygen environment. J Clin Endocrinol Metab 90:1171-1175

19. Teng CC, Tjoa S, Fennessey PV, Wilkening RB, Battaglia FC 2002 Transplacental carbohydrate and sugar alcohol concentrations and their uptakes in ovine pregnancy. Exp Biol Med (Maywood) 227:189-195

20. Parazzini F, Cortinovis I, Bortolus R, Fedele L 1991 [Standards of birthweight in Italy]. Ann Ostet Ginecol Med Perinat 112:203-246

21. Brzozowska I 1973 [Physical development parameters of newborn infants in Poland]. Probl Med Wieku Rozwoj 3:83-98

22. Battaglia FC, Meschia G 1986 An Introduction to Fetal Physiology. Academic Press, Orlando, pp 70-86

23. Lee AY, Chung SK, Chung SS 1995 Demonstration that polyol accumulation is responsible for diabetic cataract by the use of transgenic mice expressing the aldose reductase gene in the lens. Proc Natl Acad Sci USA 92:2780-2784

24. Grimshaw CE, Lai CJ 1996 Oxidized aldose reductase: in vivo factor not in vitro artifact. Arch Biochem Biophys 327:89-97

25. Panneerselvam K, Freeze HH 1996 Mannose enters mammalian cells using a specific transporter that is insensitive to glucose. J Biol Chem 271:9417-9421

26. Ogier-Denis E, Blais A, Houri JJ, Voisin T, Trugnan G, Codogno P 1994 The emergence of a basolateral 1-deoxymannojirimycin-sensitive mannose carrier is a function of intestinal epithelial cell differentiation. Evidence for a new inhibitory effect of 1-deoxymannojirimycin on facilitative mannose transport. J Biol Chem 269:4285-4290 